\title{
Severe Stevens-Johnson syndrome/toxic epidermal necrolysis overlap syndrome-beyond skin involvement
}

\author{
Tiago Milheiro Silva MD (iD | João Farela Neves MD | Ana Casimiro MD | Luís Varandas \\ PhD | Catarina Gouveia MD
}

Centro Hospitalar de Lisboa Central-EPE, Hospital Dona Estefânia, Lisbon, Portugal

\section{Correspondence}

Tiago Milheiro Silva, MD, Hospital Dona Estefânia, Centro Hospitalar de Lisboa

Central-EPE, Lisbon, Portugal.

Email: tiago.milheiro.silva@gmail.com

\begin{abstract}
Stevens-Johnson syndrome (SJS) and toxic epidermal necrolysis (TEN) are rare but serious dermatologic diseases with many potential multisystem complications. We describe the case of an 8-year-old girl who developed severe SJS/TEN overlap syndrome ( $25 \%$ of her body surface area was affected) complicated by pancreatitis and bronchiolitis obliterans. These rare complications emphasize the need for careful, intensive monitoring of possible complications and an interdisciplinary team approach to provide optimal treatment and follow-up.
\end{abstract}

\section{KEYWORDS}

drug reaction, mucous membrane disorders, pharmacology, skin barrier

\section{1 | INTRODUCTION}

Stevens-Johnson syndrome (SJS) and toxic epidermal necrolysis (TEN) are rare but serious dermatologic diseases characterized by sudden onset of high fever, signs of systemic toxicity, and a variable degree of mucocutaneous exfoliation. ${ }^{1}$ In addition to cutaneous involvement, multisystem manifestations may occur and be responsible for much of the morbidity and mortality associated with these conditions. ${ }^{1,2}$ The conjunctiva, trachea, buccal mucosa, gastrointestinal tract, and genitourinary tract are often involved. A severe and often progressive form of bronchiolitis obliterans has become an increasingly recognized complication. Pancreatic involvement has been reported in a few adult case reports. ${ }^{3-5}$ The pathophysiology has not been determined and undisputed radiologic evidence of pancreatitis in the course of SJS or TEN is lacking. ${ }^{3}$

Clinical and radiologic evidence of pancreatitis and bronchiolitis obliterans complicating a course of a severe multisystem SJS/TEN overlap in an 8-year-old child are the basis of this report.

\section{2 | CASE REPORT}

An 8-year-old girl presented to the emergency department with high-grade fever, bilateral conjunctivitis, and rash for 2 days. The parents reported having given the child paracetamol and nimesulide, a cyclooxygenase 2 selective inhibitor antiinflammatory agent, 4 days before for nasal drip and throat pain. The girl was born in India, had been in Portugal for the previous 2 years, and was being treated with iron for iron deficiency anemia for the previous 6 months. Her past medical history was unremarkable. Family history was notable for human immunodeficiency virus type1 infection in her parents and sister.

Physical examination revealed a vesicular erythematous eruption on her back, face, and arms covering approximately $10 \%$ of her body surface area (BSA). Erosions on her lips and buccal mucosa, eyelid edema, and severe bilateral exudative conjunctivitis were also noted. Initial laboratory results demonstrated lymphopenia (leukocytes 6,730/ $\mu \mathrm{L}$, lymphocytes $540 / \mu \mathrm{L}$ ) and high hepatic enzymes (aspartate aminotransferase [AST] $118 \mathrm{U} / \mathrm{L}$, normal range <35 U/L; alanine aminotransferase $[\mathrm{ALT}] 53 \mathrm{U} / \mathrm{L}$, normal range $<39 \mathrm{U} / \mathrm{L}$; gamma-glutamyl transferase [GGT] $56 \mathrm{U} / \mathrm{L}$, normal range $<22 \mathrm{U} / \mathrm{L}$; alkaline phosphatase [ALP] $265 \mathrm{U} / \mathrm{L}$, normal range 51-332 U/L) and C-reactive protein (135 $\mathrm{mg} / \mathrm{L}$, normal range $<5 \mathrm{mg} / \mathrm{L})$. Serology and molecular testing at admission and 3 weeks later were negative for viral hepatitis A, B, C, and E; Epstein-Barr virus; cytomegalovirus; herpes simplex virus; varicella-zoster virus; enterovirus; adenovirus; and Mycobacterium and Mycoplasma pneumonia. Stevens-Johnson syndrome was diagnosed and the child was transferred to an isolation room. All drugs administered before the development of clinical signs were discontinued. She was started on intravenous hydration, 
application of normal saline dressings on denuded areas, and a greasy emollient (mixture of soft and liquid paraffin) on the remaining skin and chloramphenicol for the ocular involvement.

Over the first 8 days of hospitalization there was progression of the cutaneous lesions, affecting a total of $25 \%$ of her BSA (Figure 1) with epidermal detachment of approximately $15 \%$ of her BSA, without evidence of cutaneous infection, and progressive resolution with supportive care over the next month. The mucosal lesions resolved over the 6 weeks after admission.

Ophthalmologic evaluation on day 2 revealed bilateral keratoconjunctivitis with deepithelization. Over the following weeks, bilateral corneal ulcers and symblepharon appeared and were treated using mechanical debridement and topical chloramphenicol. Three weeks after admission she developed excruciating lumbar pain accompanied by diffuse abdominal pain, especially in the epigastric area. Blood tests revealed markedly high serum amylase (600 U/L, normal range 0-31 U/L) and lipase (731 U/L, normal range 0-350 U/L) levels, mixed hyperbilirubinemia (total $1.3 \mathrm{mg} / \mathrm{dL}$, normal range $0.3-1.2 \mathrm{mg}$ / $\mathrm{dL}$; direct $1.14 \mathrm{mg} / \mathrm{dL}$, normal range 0-0.2), and high hepatic enzyme levels (AST 225 U/L, ALT 232 U/L, GGT 950 U/L, ALP 803 U/L). Abdominal sonography revealed a heterogeneous echostructure of the pancreas with preserved dimensions and evidence of biliary sludge without biliary tract dilation. Magnetic resonance cholangiopancreatography revealed edema of the medial third of the pancreatic body and dilation of the medial third of the Wirsung duct surrounded by two areas of stenosis, one in the proximal extremity and another in the area of transition to the pancreatic tail, concordant with a multifocal intrinsic stenosis process (Figure 2). A diagnosis of acute pancreatitis was made. Oral intake was stopped and intravenous fluids, analgesia, and ursodeoxycholic acid were initiated. Clinical complaints of pain subsided and a progressive normalization

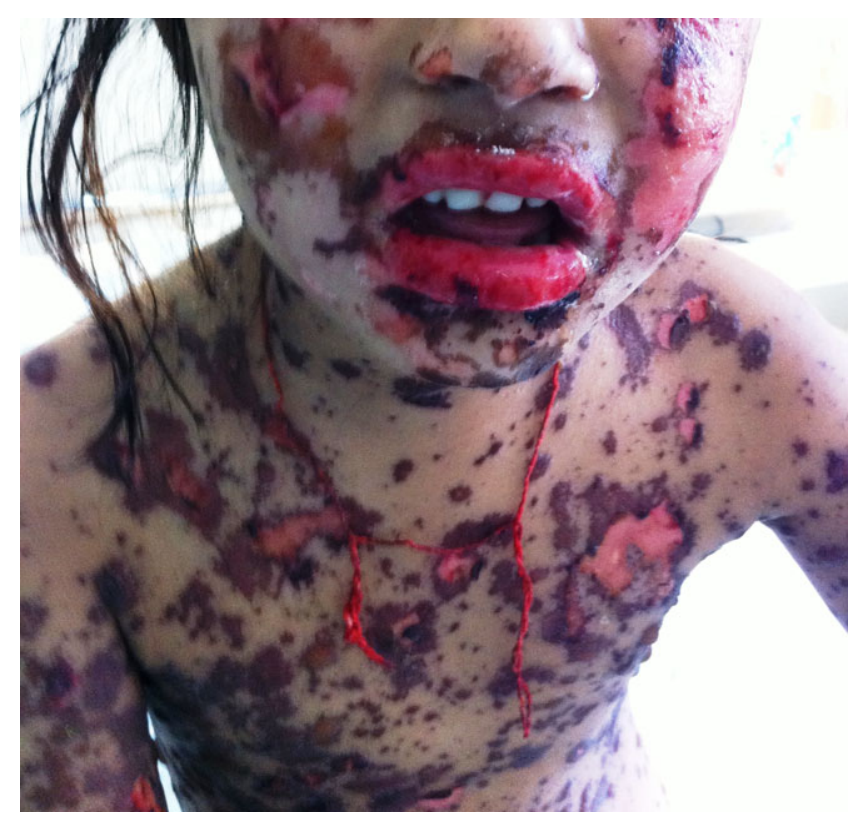

FIGURE 1 Cutaneous and mucosal lesions on the fourth day of disease

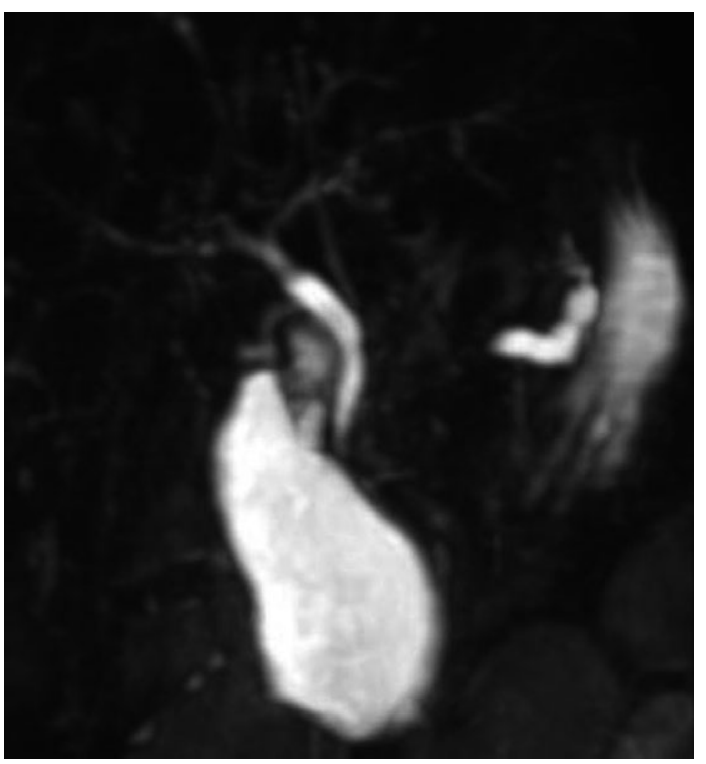

FIGURE 2 Multifocal stenosis of the Wirsung duct on cholangiopancreatic magnetic resonance imaging

of pancreatic enzymes was noted over the next 2 weeks. Oral intake was reintroduced without untoward effects.

She was febrile for a total of 36 days despite multiple antibiotics cycles. On day 34 she was treated with intravenous immunoglobulin $1 \mathrm{~g} / \mathrm{kg} / \mathrm{d}$ for 3 days, with subsequent remission of fever.

During the fifth week of hospitalization she developed progressive dyspnea and wheezing, with poor response to bronchodilators, and cervical anterior region localized pain and palpable subcutaneous emphysema. Computed tomography did not show parenchyma involvement but revealed a moderate pneumomediastinum with cervical extension. She was started on broad-spectrum antibiotics, corticosteroids, and supplemental oxygen. Over the next 2 weeks there was persistent wheezing and the need for oxygen supplementation. Pulmonary function tests revealed severe obstructive pattern with a marked increase in residual value and a lack of response to bronchodilators. Upper endoscopy and bronchoscopy were normal. A presumptive diagnosis of bronchiolitis obliterans was made and she was maintained on corticosteroid therapy for 7 days $(2 \mathrm{mg} / \mathrm{kg} / \mathrm{d})$ with subsequent slow tapering. On discharge, she was kept on a maintenance dose of $16 \mathrm{mg} / \mathrm{d}(0.7 \mathrm{mg} / \mathrm{kg} / \mathrm{d})$. Pulmonary biopsy 6 months after hospital discharge revealed peribronchiolar fibrosis with marked inflammatory changes of the pulmonary parenchyma. She was discharged 3 months after admission with complete resolution of pancreatitis, marked decrease in visual acuity, extensive areas of cutaneous hyperpigmentation, dependence on nocturnal oxygen supplementation, and maintenance corticosteroid therapy.

\section{3 | DISCUSSION}

SJS and TEN are rare but serious conditions with high morbidity and mortality often triggered by a drug or infection. ${ }^{1,5}$ Pathologically they resemble second-degree burns, with widespread keratinocyte 
necrosis in all epidermal layers. ${ }^{5}$ In addition to cutaneous involvement, mucosal lesions are frequently reported, causing significant long-term morbidity. ${ }^{1}$ Dysfunction of various organ systems, including renal, respiratory, and digestive, may also occur. ${ }^{1,3}$

Pancreatitis occurring in individuals with SJS and TEN is well known but is not a common finding. ${ }^{3,4,6} \mathrm{~A}$ study by Dilewski and colleagues ${ }^{6}$ demonstrated that $40 \%$ of individuals with SJS/TEN developed asymptomatic elevations of amylase and lipase levels. Coetzer et al. ${ }^{3}$ reported a 25 -year-old man with TEN who, like our patient, developed clinical evidence of pancreatitis, with high serum amylase levels and pancreatic swelling and biliary sludge without biliary tract dilatation on ultrasonography. Our patient also had Wirsung duct stenosis, as evidenced by magnetic resonance cholangiopancreatography. The mechanisms of pancreatitis in these conditions are unclear. ${ }^{3}$ An immunologic pathway of pancreatic damage or hypovolemia is thought to be the most plausible mechanism. ${ }^{3}$ Although treatment of pancreatitis is typically fluid restriction and supportive care, the myriad and timing of complications in our patient prompted us to initiate corticosteroid and immunoglobulin therapy.

Individuals with SJS and TEN have high caloric and protein requirements, and appropriate nutrition is necessary to increase survival. ${ }^{6}$ Children are even more vulnerable to suboptimal nutrition because they have low lean body mass reserves. ${ }^{7}$ The diagnosis of pancreatitis impeded the initiation of enteral nutrition, which might have worsened the prognosis in our patient. ${ }^{8}$

Bronchiolitis obliterans has also been reported in SJS/TEN. It is characterized by partial or complete obstruction of the bronchioles or alveolar ducts by granulation tissue or fibrosis. ${ }^{9}$ The onset of bronchiolitis obliterans has been reported to occur 5 days to 10 months after the development of SJS. ${ }^{10}$ Our patient presented with dyspnea 1 month after the onset of skin lesions. Although the pulmonary biopsy findings were nonspecific, the peribronchial fibrosis and the severe obstructive pattern, with no response to bronchodilators, were consistent with the diagnosis of bronchiolitis obliterans. There is no consensus as to the optimal treatment for bronchiolitis obliterans. ${ }^{11}$ Although systemic corticosteroids are frequently used, their use is controversial, and large-scale studies are lacking regarding optimal treatment and prognosis in bronchiolitis obliterans associated with SJS/TEN. ${ }^{11}$

Management of SJS/TEN requires careful, intensive monitoring of possible multisystem complications and an interdisciplinary team approach to provide optimal care and follow-up. ${ }^{1}$

Given the rarity of these conditions, large, multicenter, prospective studies are required to establish best practices for management and to improve outcomes with these life-threatening diseases.

\section{ORCID}

Tiago Milheiro Silva (iD http://orcid.org/0000-0003-1170-5674

\section{REFERENCES}

1. Gerull R, Nelle M, Schaible T. Toxic epidermal necrolysis and Stevens Johnson syndrome: a review. Crit Care Med. 2011;39:1-12.

2. Finkelstein $\mathrm{Y}$, Soon GS, Acuna P, et al. Recurrence and outcomes of Stevens-Johnson syndrome and toxic epidermal necrolysis in children. Pediatrics. 2011;128:723-728.

3. Coetzer M, van der Merwe AE, Warren BL. Toxic epidermal necrolysis in a burn patient complicated by acute pancreatitis. Burns. 1998;24:181-183.

4. Chosidow O, El Wady Z, Devanlay M, et al. Hyperamylasemia in toxic epidermal necrolysis. Arch Dermatol. 1993;129:792-793.

5. Wetter DA, Camilleri MJ. Clinical, etiologic, and histopathologic features of Stevens Johnson syndrome during an 8-year period at Mayo Clinic. Mayo Clin Proc. 2010;85:131-138.

6. Dylewski ML, Prelack K, Keaney T, Sheridan RL. Asymptomatic hyperamylasemia and hyperlipasemia in pediatric patients with toxic epidermal necrolysis. J Burn Care Res. 2010;31:292-296.

7. Harmel RP, Vane DW, King DR. Burn care in children: special circumstances. Clin Plast Surg. 1986;13:95-105.

8. Park AJ, Latif SU, Ahmad MV, et al. A comparison of presentation and management trends in acute pancreatitis between infants/toddlers and older children. J Pediatr Gastroenterol Nutr. 2010;51:167170.

9. Kim MJ, Lee KY. Bronchiolitis obliterans in children with StevenJohnson syndrome: follow up with high resolution CT. Pediatr Radiol. 1996;26:22-25.

10. Pannu BS, Egan AM, Lyer VN. Phenytoin induced Stevens Johnson syndrome and bronchiolitis obliterans: case report and review of literature. Respir Med Case Rep 2016;17:54e56.

11. Tomikawa SO, Adde FV, Ferreira da Silva Filho LV, Leone C, Rodrigues JC. Follow-up on pediatric patients with bronchiolitis obliterans treated with corticosteroid pulse therapy. Orphanet J Rare Dis 2014;9:128.

How to cite this article: Milheiro Silva T, Farela Neves J, Casimiro A, Varandas L, Gouveia C. Severe Stevens-Johnson syndrome/toxic epidermal necrolysis overlap syndromebeyond skin involvement. Pediatr Dermatol. 2018;35:e17-e19. https://doi.org/10.1111/pde.13328 